\title{
The LOFAR Tied-Array All-Sky Survey for Pulsars and Fast Transients
}

\author{
C. M. $\operatorname{Tan}^{1}$ and the LOTAAS Group ${ }^{2}$ \\ ${ }^{1}$ Jodrell Bank Centre for Astrophysics, University of Manchester \\ Oxford Road, Manchester M13 9PL, UK \\ email: chiamin.tan@postgrad.manchester.ac.uk \\ ${ }^{2}$ C. G. Bassa, S. Cooper, J. W. T. Hessels, V. I. Kondratiev, D. Michilli, S. Sanidas, \\ B. W. Stappers, S. ter Veen, J. van Leeuwen
}

\begin{abstract}
The LOFAR Tied Array All-Sky Survey (LOTAAS) is an ongoing all northern sky survey for pulsars and transients. It is one of the first large scale pulsar surveys conducted at an observing frequency below $200 \mathrm{MHz}$. The unique set-up of the survey is the simultaneous formation of 222 beams for each survey pointing by coherently adding signals from the central 6 LOFAR stations. This represents the first SKA-like pulsar survey. As of 12 September 2017, the survey has completed 1456 pointings, more than two-thirds of the total. The survey has discovered 61 new pulsars via Fourier-based periodicity searches and a further 5 via single pulse searches. I present the survey approach and distinctive features including a discussion of an improved machine learning classifier used to identify the best candidates produced by the pipeline for further investigation. I present a summary of the discoveries so far including the first binary pulsar and the pulsar with the longest spin period of $23.5 \mathrm{~s}$.
\end{abstract}

Keywords. pulsars: general, surveys, methods: data analysis

\section{Introduction}

Since the first discovery of pulsars exactly 50 years ago (Hewish et al. 1968), various large scale surveys have been conducted by telescopes all over the world to increase the known sample. This has resulted in the discovery of more than 2500 pulsars. Although the first pulsar was discovered at a low observing frequency of $81 \mathrm{MHz}$, most pulsar surveys are conducted at higher observing frequencies of $>400 \mathrm{MHz}$. This is primarily to avoid the effects of the interstellar medium (ISM), including dispersion where the propagation of radio signals are delayed by the free electrons in the ISM, as well as pulse broadening due to scattering in the ISM. Both of these effects are more pronounced at low observing frequencies (Lorimer \& Kramer 2005). However, with improvements in sensitivity and processing capability there is a resurgence of pulsar surveys conducted at low observing frequencies, allowing us to take advantage of the steep spectrum nature of pulsars (Maron et al. 2000, Bates et al. 2013, Bilous et al. 2015). One of the major pulsar surveys at low frequency is the LOFAR Tied-Array All-Sky Survey (LOTAAS).

LOFAR (LOw Frequency ARray, Stappers et al. 2011, van Haarlem et al. 2013) is an interferometric array consisting of thousands of dipole antennas spread across the Netherlands and several other European countries. Two different types of antennas are used to cover the observing frequency range of $10-240 \mathrm{MHz}$; the low-band antennas (LBAs) covering frequency range of $10-90 \mathrm{MHz}$ and the high-band antennas (HBAs) covering 110-240 MHz. LOTAAS utilizes the HBAs for the survey. This article provides a brief description of LOTAAS and a summary of the discoveries it has made. A more in-depth description of the survey will be discussed in Sanidas et al. (in prep). 


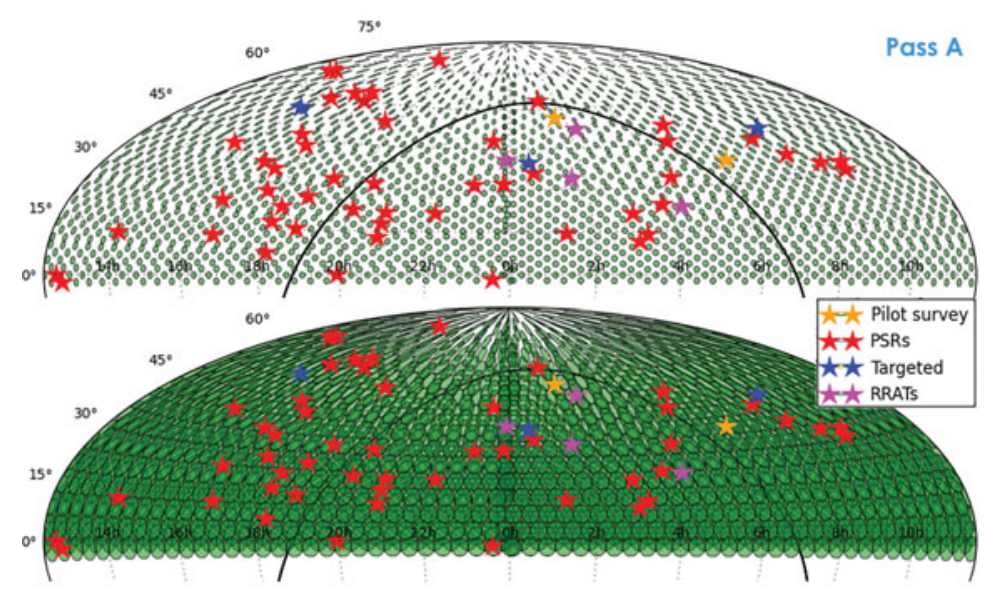

Figure 1. The sky coverage of the TABs (top) and incoherent beams (bottom) by the first pass of LOTAAS, overlaid with the position of LOTAAS discoveries.

\section{Survey Details}

\subsection{Survey Setup and Progress}

LOTAAS uses 12 HBA sub-stations of LOFAR located on the Superterp, a circular area of $320 \mathrm{~m}$ diameter where the concentration of stations of the array is the highest. Each LOTAAS pointing has a bandwidth of $32 \mathrm{MHz}$, centred at $135 \mathrm{MHz}$ and a dwell time of 1 hour. The data is recorded with 2592 channels of $12 \mathrm{kHz}$ bandwidth each and a sampling time of $492 \mu \mathrm{s}$. A total of 222 individual beams are produced for each LOTAAS pointing, split into 3 sub-array pointings (SAPs), directed at 3 nearby positions on the sky. An incoherent beam is formed for each of the three SAPs by incoherently adding the signals from the 12 sub-stations. These beams have a full-width half-maximum of $5.5^{\circ}$ at $135 \mathrm{MHz}$. A hexagonal grid of 61 tied-array beams (TABs) is formed by coherently adding the station beams and placed at the centre of each SAP. The individual TABs have a full-width half-maximum of $0.4^{\circ}$ at $135 \mathrm{MHz}$. A further 12 TABs are formed for each SAP and these are directed towards any known pulsar within the field-of-view of the SAP that is not covered by the hexagonal grid, or towards a pre-determined position if there are no known sources.

A total of 3 passes of 651 pointings each is planned for the full LOTAAS survey in order to cover the whole northern sky with TABs. As of 12 September 2017, the first pass of the survey has been completed, covering the whole northern sky with the incoherent beams (See Figure. 1). A further 650 of the 651 pointings of the second pass are completed and 155 pointings have been observed for the third pass.

\subsection{Data Processing}

The raw data obtained from the survey are stored in the LOFAR long term archive, hosted by ASTRON. The data are then transferred to the Dutch National Supercomputer Cartesius $\dagger$ for processing. The data from each beam are processed individually on a single Cartesius CPU node which has 24 cores and memory of 64 GB. A PRESTO (Ransom et al. 2002) based search pipeline is used to process the data. The data is first dedispersed with various trial $\mathrm{DM}$ values ranging from 0 to $550 \mathrm{pc} \mathrm{cm}^{-3}$. The dedispersed time series are then searched for pulsars using two different methods; a Fourier based periodicity search where a Fast Fourier Transform is applied to the dedispersed time series to search for

$\dagger$ https://userinfo.surfsara.nl/systems/cartesius 
periodic signals and a single pulse search where the dedispersed time series is searched for significant events. Diagnostic plots of pulsar candidates from both search methods are then produced. The search pipeline on a single beam on requires on average three hours of processing time on a single node.

\subsection{Candidate Selection}

The periodicity search part of the processing pipeline yields an average of 20,000 candidates for each LOTAAS pointing processed. At this rate, we are expecting 40 million candidates by the end of the survey. The number of candidates produced would be too large for visual inspection. Hence, we used Machine Learning (ML, Bishop 2006) to develop classifiers capable of identifying candidates that are most likely to be pulsars. The first ML classifier extracts 8 features from the integrated pulse profile and DM curve of the diagnostic plots of the candidates (Lyon et al. 2016), trained with a sample of pulsars and non-pulsars by Cooper (2017). A very fast decision tree (VFDT, Quinlan 1993) algorithm is used to produce a binary classifier. The ML classifier produced is able to reduce the number of candidates to inspect per pointing from 20,000 to about 500, with a recall rate for pulsars of 96.2 per cent.

Although the first ML classifier is highly successful at identifying new pulsars, we found out that the classifier is less accurate for pulsars with wide integrated pulse profiles. To overcome this, we implemented several improvements and produced a new ML classifier. These include the introduction of 12 new features, in which 8 of them are extracted from the pulse intensity as a function of time and phase, along with the pulse intensity as a function of sub-band and phase plots. The other 4 new features are extracted from the DM curve. A third class of candidates, consisting of known RFI instances in the data is introduced to the classifier. The ML classifier is produced by combining 5 VFDT classifiers, each trained with a different training dataset. The new classifier is able to further reduce the number of candidates for visual inspection to about 220 candidates per pointing, with an improved recall rate for pulsars of 98.7 per cent. More importantly, the new classifier is able to identify pulsars with wide integrated pulse profiles with higher accuracy. A detailed description of the new ML classifier can be found in Tan et al. (2017, submitted).

The single pulse search pipeline generated events on the order of $10^{8}$ for each pointing processed. Michilli et al. (in prep.) developed a single pulse classifier that combines two approaches to identify promising candidates. The first approach involves various standard methods to reduce the number of candidates, including event grouping in DM-time space, removal of low-DM events and comparison of events between different TABs of the same pointing. The second approach involves an ML classifier that uses 5 features extracted from the grouped events to identify them as either pulsars or RFI. The single pulse classifier is able to reduce the number of candidates to roughly 20 per pointing. Diagnostic plots are then generated for these candidates for visual inspection. The single pulse classifier identifies 8 of the LOTAAS discovery and a further 80 known pulsars.

\section{Discoveries}

As of 12 September 2017, LOTAAS has discovered 66 new pulsars, includes 5 rotating radio transients (RRATs). The discoveries can be found on the LOTAAS website $†$. The LOTAAS discoveries that are confirmed by follow-up observations are then added to the LOFAR timing program. The LOTAAS discoveries are also being observed by the

$\dagger$ http://www.astron.nl/lotaas 
Lovell Telescope at an observing frequency of $1.4 \mathrm{GHz}$ and timed if they are detected. Currently about half of the pulsars discovered by LOTAAS are not detected by the Lovell Telescope, suggesting that a significant fraction of the population of pulsars discovered could have large spectral indices.

PSR J1658+36 is a binary pulsar that was first discovered by reclassifying candidates from archive pointings with the new ML classifier. The pulsar has a spin period of $33 \mathrm{~ms}$ and a DM of $3 \mathrm{pc} \mathrm{cm}^{-3}$. Several follow-up observations of the pulsar revealed that it is in a binary system of orbital period of 3.0 days with a companion that has a minimum mass of $0.87 \mathrm{M}_{\odot}$. Searches of archival optical images at the position of the pulsar revealed a possible white dwarf companion. The pulsar is observed but not detected by the Lovell Telescope at observing frequencies of $350 \mathrm{MHz}$ and $1.4 \mathrm{GHz}$.

More recently, PSR J0250+58, a pulsar with a spin period of $23.5 \mathrm{~s}$, the longest spin period of any of the known pulsars was discovered. The pulsar has a DM of $45 \mathrm{pc} \mathrm{cm}^{-3}$. Several follow up observations allowed us to confirm the periodicity and localize the pulsar. The pulsar is shown to have a duty cycle of 0.4 per cent and the pulse intensity varies over 1 hour, with little variation in pulse shape.

\section{Conclusion and Future}

LOTAAS is currently one of the deepest and most successful low frequency pulsar surveys ever performed. It has now discovered more than 60 pulsars, including the pulsar with the longest spin period. With the current rate of discovery, we expect to uncover about 100 pulsars by the end of the survey. Discovering new pulsars is only half of the survey goal of LOTAAS. In order to understand the population of pulsars better, regular timing of the pulsars are required and will be achieved by the timing campaign by both LOFAR and the Lovell telescope.

Several improvements in the survey are planned for the near future. A new processing pipeline is currently being developed which will include a Fast Folding Algorithm (FFA) that is more sensitive to long period pulsars and/or pulsars with small duty cycle, similar to $\mathrm{J} 0250+58$. Next, we plan to increase the time resolution of the survey from $492 \mu \mathrm{s}$ to either $246 \mu \mathrm{s}$ or $164 \mu \mathrm{s}$. Finally, we plan to extend the sky coverage of the survey up to a declination of $-10^{\circ}$.

\section{References}

Bates, S. D., Lorimer, D. R., \& Verbiest, J. P. W.. 2013, MNRAS, 431, 1352

Bilous, A., et al. 2015, A\&SA, 591, A134

Bishop, C. M. 2006, Pattern Recognition and Machine Learning, Springer

Cooper. S. 2017, PhD Thesis, University of Manchester

Hewish, A., Bell, S. J., Pilkington, J. D. H.., Scott, P. F., \& Collins, R. A. 1968, Nature, 217, 709

Lorimer, D. R. \& Kramer, M. 2005, Handbook of Pulsar Astronomy, Cambridge University Press

Lyon, R. J., Stappers, B. W., Cooper, S., Brooke, J. M., \& Knowles, J. D. 2016, MNRAS, 459

Maron, O., Kijak, J., Kramer, M., \& Wielebinski, R. 2000, A\&̊AS, 147, 195

Quinlan, J. R. 1993, C4.5: programs for machine learning, Morgan Kaufmann

Ransom, S. M., Eikenberry, S. S., \& Middleditch, J. 2002, AJ, 124

Stappers, B. W., et al. 2011, A\& $A, 530, \mathrm{~A} 80$

van Haarlem, M. P., et al. 2013, A\&GA, 556, A2 